\title{
RELATIONS AMONG PARAMETERS ALONG THE RATIONAL CUBIC CURVE.
}

BY PROFESSOR J. E. ROWE.

(Read before the American Mathematical Society, April 24, 1915.)

\section{Introduction.}

The purpose of this paper is to give the proofs of two new theorems concerning relations among sets of parameters along the rational plane cubic curve. The first theorem concerns a projective relation possessed by the parameters of the four residual points in which the osculant conic at any point meets the cubic. The second theorem defines the relation which exists among the parameters of the four tangents drawn from any point of the plane to the rational cubic. The proof depends upon a method of deriving the parametric equations of the node of the rational cubic.

We shall call the rational plane cubic the $R^{3}$, and write its parametric equations in the form

$$
x_{i}=\left(a_{i} t\right)^{3} \equiv a_{i} t^{3}+3 b_{i} t^{2}+3 c_{i} t+d_{i} \quad(i=0,1,2) .
$$

$\S 1$. The Osculant Conic and the Associated Theorem

A point on the osculant conic of the $R^{3}$ at a point whose parameter is $t^{\prime}$ is defined by the equations

$$
\begin{aligned}
x_{i}=\left(\alpha_{i} t^{\prime}\right)\left(\alpha_{i} t\right)^{2} \equiv\left(a_{i} t^{\prime}+b_{i}\right) t^{2} & +2\left(b_{i} t^{\prime}+c_{i}\right) t \\
& +\left(c_{i} t^{\prime}+d_{i}\right) \quad(i=0,1,2) .
\end{aligned}
$$

The equation* of the osculant conic at $t$ has the form

$$
\begin{aligned}
& {\left[4|a b x||b c x|-|a c x|^{2}\right] t^{4}+[4|a b x||b d x|-2|a c x||a d x|} \\
& \quad+2|a c x||b c x|] t^{3}+(\cdots) t^{2}+(\cdots) t+(\cdots)=0 .
\end{aligned}
$$

In particular, if $t=0$, (3) becomes

$$
4|b c x||c d x|-|b d x|^{2}=0 .
$$

If the values of $x_{i}$ from (1) are substituted in (4), and the factor $t^{2}$ is removed, the result is

$$
\begin{aligned}
{[4|a b c||a c d|} & \left.-|a b d|^{2}\right] t^{4}+12|a b c||b c d| t^{3} \\
& +6|a b d||b c d| t^{2}+4|a c d||b c d| t+3|b c d|^{2}=0 .
\end{aligned}
$$

* J. E. Rowe, Messenger of Math., No. 512 (Dec., 1913), pp. 118-119. 
The roots of (5) are the parameters of the residual points in which the conic (4) cuts the $R^{3}$. The invariant $S$ of (5) is

(6) $12|a b c||a c d||b c d|^{2}-3|a b d|^{2}|b c d|^{2}-12|a b c||a c d||b c d|^{2}$

$$
+3|a b d|^{2}|b c d|^{2}
$$

which vanishes identically. No restriction was imposed by selecting the osculant conic of the $R^{3}$ at the point whose parameter is 0 ; i. e., if $S=0$ for (5) derived from this particular osculant conic, the same projective relation $S=0$ will exist for every quartic found by selecting a particular value for $t$ in (3). This result may be stated in the form of

Theorem I. The parameters of the four residual points of intersection of the $R^{3}$ and any osculant conic form a self-apolar set.

\section{$\S 2$. The Equation of the Node of the $R^{3}$ and its Significance.}

Every line section of the $R^{3}$ has three parameters which are apolar to the three flex parameters. The two nodal parameters and any other are apolar to the three flex parameters, for any line on the node determines the two nodal parameters and one other; this third parameter may be used to distinguish one line on the node from another.

From any point in the plane four tangents can be drawn to the $R^{3}$. The four parameters of the tangents from the point $x_{i}$ to the $R^{3}$ are the roots of the quartic*

$$
\begin{aligned}
(\alpha t)^{4} \equiv|a b x| t^{4}+2|a c x| t^{3}+[a d x+ & 3|b c x|] t^{2} \\
& +2|b d x| t+|c d x|=0 .
\end{aligned}
$$

The three flex parameters $\dagger$ are the roots of

$$
(\beta t)^{3} \equiv|a b c| t^{3}+|a b d| t^{2}+|a c d| t+|b c d|=0 .
$$

The two nodal parameters may be found as the pair which, together with an arbitrary third, are apolar to (8); this amounts to finding the Hessian of (8). Hence, they are given by

$$
H \equiv\left|\begin{array}{ccc}
3|a b c| & |a b d| & |a c d| \\
|a b d| & |a c d| & 3|b c d| \\
1 & -t & t^{2}
\end{array}\right|=0
$$

* Loc. cit., p. 119.

$\dagger$ For equation (8) and its Hessian $H$ see Salmon's Higher Plane Curves, third edition, pp. 187-188. 
The polar of (8) with respect to (7) is, in symbols,

$$
(\beta \alpha)^{3}(\alpha t)=0,
$$

which expanded becomes

$$
\begin{aligned}
& {[3|a b c||b d x|-|a b d|(|a d x|+3|b c x|)+3|a c d||a c x|} \\
& -6|b c d||a b x|] t+[6|a b c||c d x|-3|a b d||b d x| \\
& +|a c d|(|a d x|+3|b c x|)-3|b c d||a c x|]=0 .
\end{aligned}
$$

For a given value of $t,(10)$ is the equation of a line; the intersections of this line and the $R^{3}$ may be found by substituting from (1) in (10). If $t=t_{1}$ in (10) and the values of $x_{i}$ from (1) are substituted in the equation of the line so derived, the result may be thrown into the form

$$
H\left(t-t_{1}\right)=0 \text {. }
$$

This is sufficient to show that (10) is a pencil* of lines on the node, and may be said to envelope the node. The parametric equations of the node as a rational curve of the first class are found by identifying (10) and the equation

$$
(\xi x)=\xi_{0} x_{0}+\xi_{1} x_{1}+\xi_{2} x_{2}=0 ;
$$

these assume the form

$$
\begin{aligned}
\xi_{i}= & {\left[3|a b c|\left(b_{\mu} d_{\nu}\right) \cdots-6|b c d|\left(a_{\mu} b_{\nu}\right)\right] t } \\
& +\left[6|a b c|\left(c_{\mu} d_{\nu}\right) \cdots-3|b c d|\left(a_{\mu} c_{\nu}\right)\right] \quad(i, \mu, \nu=0,1,2) .
\end{aligned}
$$

Also (10) as it stands may be considered the equation of the node. This shows how the parameters of the four tangents from any point of the plane are related. The equation (10) for a particular set of the $x_{i}$ yields a value of $t$ which together with the three flex parameters constitutes a set of four apolar to the quartic found by substituting the set of the $x_{i}$ in (7). The result may be stated as a theorem.

Theorem II. The parameters of the four tangents from any point $P$ of the plane to the $R^{3}$ are apolar to the set of four composed of the three flex parameters and the parameter of the other point cut out of the $R^{3}$ by the line joining $P$ to the node of the $R^{3}$.

Pennsylvania State College, March, 1915.

* Compare with W. Gross, Mathematische Annalen, vol. 32 (1888), pp. 144-145. 